\title{
Artelogie
}

artelogie Recherche sur les arts, le patrimoine et la littérature de l'Amérique latine

$13 \mid 2019$

Violeta Parra: authenticité, primitivisme et processus d'exotisme chez les artistes latino-américains.

\section{Imaginaires de l'espace fluvial : aspects d'une géocritique de l'Orénoque}

\section{Karolina Katsika}

\section{CpenEdition}

1 Journals

Édition électronique

URL : http://journals.openedition.org/artelogie/3409

DOI : 10.4000/artelogie.3409

ISSN : 2115-6395

Éditeur

Association ESCAL

Référence électronique

Karolina Katsika, «Imaginaires de l'espace fluvial : aspects d'une géocritique de l'Orénoque », Artelogie [En ligne], 13 | 2019, mis en ligne le 07 janvier 2019, consulté le 20 avril 2019. URL : http:// journals.openedition.org/artelogie/3409; DOI : 10.4000/artelogie.3409

Ce document a été généré automatiquement le 20 avril 2019.

Association ESCAL 


\title{
Imaginaires de l'espace fluvial : aspects d'une géocritique de l'Orénoque
}

\author{
Karolina Katsika
}

1 Depuis sa « découverte » et son « exploration » par les Européens, l'Orénoque, fleuve qui traverse le Venezuela et la Colombie, a longtemps constitué un espace quasi mythique en Europe. Espace impressionnant par sa faune, dont les fameux piranhas et les anacondas, sa flore et ses paysages imposants, espace "sauvage » et "vierge ", l'Orénoque tient sa place dans la littérature francophone. En effet, qualifié tour à tour de mystérieux, de majestueux ou d'effrayant, tout un imaginaire ambivalent s'est construit autour de l'espace fluvial de l'Orénoque. Et si aujourd'hui le Delta de l'Orénoque est évalué en tant que destination touristique sur Tripadvisor, le fleuve continue à alimenter l'imaginaire littéraire. Nous nous interrogerons donc sur la construction et l'évolution de l'imaginaire de l'Orénoque. Après un détour par les écrivains du XIXe siècle (Jules Crevaux, Jules Verne), nous explorerons les représentations de cet espace fluvial dans l'œuvre Moravagine (1926) de l'écrivain suisse Blaise Cendrars et dans Remonter l'Orénoque (2005) de l'écrivain français Mathias Enard. Presqu'un siècle sépare les deux œuvres, ce qui nous permettra d'identifier, par une approche comparatiste, les mutations de la perception de l'espace fluvial ainsi que son rôle dans la narration. Par ailleurs, selon B.Westphal, les représentations de l'espace référentiel, réel se retrouvent dans l'espace fictionnel, car en l'occurrence l'espace littéraire ne se réfère pas à un espace abstrait, conceptuel mais à un lieu concret, factuel, un réalème,. Il existe en effet une "relation entre espaces référentiels et espaces fictionnalisés ni totalement séparés, ni totalement confondus ", une « relation variable » (WESTPHAL, 2007 : 17-18) entre lieu fictionnel et lieu réel. Nous aborderons donc, par une approche géocritique, l'espace fictionnel de l'Orénoque qui prend ses libertés - ou pas - par rapport à l'espace réel.

2 Nous nous pencherons dans un premier temps sur deux œuvres assez caractéristiques du XIXe siècle qui se déroulent dans l'espace fluvial de l'Orénoque : le récit de voyage $E n$ radeau sur l'Orénoque. Des Andes aux bouches du Grand Fleuve écrit par le médecin militaire et 
explorateur Jules Crevaux et le roman d'aventures Le Superbe Orénoque de Jules Verne. À travers l'analyse du système descriptif et du récit, nous tenterons de relever les constantes qui forment l'image du fleuve.

3 Les deux auteurs font une large part à la flore qui pousse autour de l'Orénoque. Tous deux mentionnent la sarrapia "très employée en parfumerie, et comme succédané de la quinine » (CREVAUX, $1882: 166$ ). Cependant si, chez Jules Verne, les différentes plantes, telles que la fève tonka ou le balsame forment en quelque sorte partie du décor tropical, Jules Crevaux mentionne les plantes par leur nom scientifique en citant « les mauvaises herbes du pays : sidées, convolvulacées, cucurbitacées» (ibid: 177) et en collectionnant des échantillons. Les lexèmes de botanique mettent ainsi en valeur sa propre identité de scientifique. Il en va de même de l'exotisme animalier: dans Le Superbe Orénoque, les animaux sauvages mentionnés, comme les oiseaux aquatiques - canards sylvestres, guanaguanares, gabiotas - construisent un exotisme qui met en avant l'inconnu, l'original, tandis que dans En radeau sur l'Orénoque, sont minutieusement notées les différentes espèces de tortues qui vivent dans l'Orénoque, « tortue de l'Orénoque (tortuga ); térékaï ; une autre tortue, la cabesona ; une dernière espèce, sorte de térékaï, beaucoup plus petit» (ibid : 169). Au-delà de la curiosité que suscite la faune exotique, celle-ci ne manque pas d'inspirer la peur et l'effroi. Tout d'abord elle participe aux conditions extrêmes où vivent les voyageurs. Les personnages font face à des «ces légions de moustiques, qui rendent l'endroit inhabitable, ces myriades d'insectes", une "race maudite » (VERNE, 1898), sont «harcelés tout le jour par des nuées de pions », ont des puces chiques sous les ongles, et la nuit doivent dormir sur des hamacs afin d'éviter les serpents et sous des moustiquaires afin de se protéger des "innombrables légions de diptères agaçants" et de "leurs appétits dévorants " (CREVAUX: 158, 204). Plus dangereux, les caïmans qui n'hésitent pas à attaquer les navigateurs: Apatou qui fait partie de l'équipage de l'expédition de Crevaux se voit arracher une partie de sa jambe par un de ces crocodiliens. Dans Le Superbe Orénoque, défilent « ces fauves, habitués des vastes campagnes vénézuéliennes, des jaguars, des pumas, des tigres, des ocelots, non moins redoutables que s'ils eussent librement couru la forêt ou la plaine. Et c'était contre ces bandes que se défendaient deux hommes, à coups de fusil et de revolver » (VERNE). L'Orénoque semble donc être un espace de nature sauvage et de péril mortel, un espace inquiétant, qui inspire la terreur et éveille la peur ancestrale de la dévoration. Allant plus loin, Verne met en scène une "innombrable et irrésistible armée de grandes tortues arrau » qui provoque presque un séisme, ces chéloniens entrainnant tout sur leur passage et «portant sur elles des escadrilles de singes effrayés, de tigres» (ibid.). Si la séquence manque de vraisemblance, ce qui est une caractéristique du roman d'aventures, elle participe tout de même à la construction d'une image du fleuve en tant qu'espace de l'extraordinaire, du merveilleux. Par ailleurs, la narration opère des stases descriptives des paysages fluviaux. Le narrateur de En radeau sur l'Orénoque, remarque que "l'Orénoque, extrêmement large, est ici semé d'îles bordées de bancs de sables. [...] La végétation des rives manque de grandeur; c'est du fouillis; pas de troncs géants comme sur les bords du Guaviare; partout des arbres étriqués, étouffés presque » (CREVAUX: 178). Si cette description à focalisation interne est quelque peu déceptive par rapport aux attentes de splendeur de la part du voyageur, celle du delta de l'Orénoque fait état des «mille bars du fleuve [qui] s'anastomosent en réseau serré à travers une végétation luxuriante » (ibid. : 186). Le vocabulaire de physique et de chirurgie souligne encore une fois la focalisation interne du médecin voyageur et, par là, la fonction mathésique de la description qui est mise en avant. Cette fonction mathésique, encore une constante des 
récits de voyage, apparait également dans Le Superbe Orénoque, où le narrateur mentionne le " "fleuve de llanos", ces vastes surfaces propices à toutes cultures, si heureusement disposées pour l'élevage des bestiaux » (VERNE). Ou bien, afin de désigner les courants rapides, l'auteur utilise le lexème local de «raudal » en l'expliquant : «ce raudal est une sorte de couloir, creusé entre les montagnes escarpées de la rive, d'une longueur de dix kilomètres. Les eaux, irritées par le resserrement du défilé où leur pente les engage, deviennent torrentueuses " (ibid.). Le léxème étranger participe à la construction d'un espace exotique, étrange tout en ayant un rôle de transmetteur de savoir. La connaissance du lieu se fait également par d'autres moyens diégétiques :

- Monsieur Miguel, nous voici mouillés à l'embouchure du Meta....

- Effectivement, monsieur Helloch.

- C'est un des affluents de l'Orénoque ?...

- Oui, et l'un des plus importants, puisqu'il lui verse quatre mille cinq cents mètres

cubes d'eau par seconde. (ibid.)

C'est par le dialogue, acquérant une fonction mathésique, entre un savant et un explorateur que le narrataire reçoit les informations géographiques sur le fleuve et ses affluents. Les interrogations brèves et successives témoignent non seulement d'une envie de savoir mais aussi d'une certaine préoccupation, d'un certain émoi. Le fleuve apparaît alors comme un espace inconnu, voire mystérieux et obscur. Et de là, il devient un lieu fantomatique : les voyageurs entendent des sons insolites, «leur oreille avait été frappée par une suite de sons musicaux très distincts, un ensemble harmonique d'une intensité particulière ", ils « auraient pu se croire à Thèbes, au pied de la statue de Memnon [...] Au lever du soleil, dit-il, cette musique que perçoivent nos oreilles, eût été plus perceptible encore, et voici quelle en est la cause. Ces roches contiennent en grand nombre des paillettes de mica. Sous les rayons solaires, l'air dilaté s'échappe des fissures de ces roches, et, en s'échappant, fait vibrer ces paillettes » (ibid.). Autant le phénomène que son explication semblent étonnants, faisant de l'Orénoque un espace féérique, irréel. Par ailleurs, la référence à la statue du colosse de Memnon à Thèbes, en Égypte antique qui selon Strabon, se mettait à fredonner à chaque aube, marque le passage par l'héritage gréc, et donc européen, et par conséquent le passage par le connu afin d'appréhender l'inconnu et d'aménuiser son étrangeté. De la même manière, Crevaux note que le " fleuve est large et houleux; les vagues moutonnent comme en mer " (CREVAUX : 165). La métaphore maritime insère le familier dans l'exotique. En outre, comme le remarque C. Lepagnez, le personnage principal du Superbe Orénoque, Jean de Kermor et son oncle, le sergent Martial, étant originaires de Nantes - tout comme Jules Verne - , « le comparant naturel de l'Orénoque est la Loire » (LEPAGNEZ, 1998 : 38-39). En effet, et c'est encore par un dialogue que la ressemblance est relevée, les deux personnages discutent :

Alors le sergent Martial d'ajouter :

- Est-ce que, par hasard, nous sommes revenus dans notre cher pays de Bretagne?

....

- Je te comprends, répondit Jean. Ici, l'Orénoque ressemble à la Loire... (VERNE)

5 La comparaison revient également sous forme de description: "Les trois falcas marchaient de conserve, sensiblement animées de la même vitesse, tantôt en file comme les chalands de la Loire "(ibid.). La coexistence dans la même phrase de l'exotisme langagier du terme falcas et du toponyme familier, marque le rôle rassurant de ce dernier face au paysage singulier.

Les populations locales font également partie du paysage fluvial et font donc l'objet de descriptions diverses. Si, dans Le Superbe Orénoque, le descriptif tourne essentiellement 
autour du physique et de l'habillement des autochtones, insistant sur les détails singuliers, comme leur peau teintée de rouge végétal, dans En radeau sur l'Orénoque, Cravaux diagnostique les maladies dont souffrent les Indiens, décrit leurs plaies, leurs pathologies, leurs infections. Cependant le plus souvent il n'est pas question de les soigner, mais de se contenter à l'observation. Selon E. Lézy, « lui qui rêvait de répandre les bienfaits de la civilisation, de soigner sur son passage l'ignorance et la maladie, doit bientôt constater que son contact est plus mortifère que salutaire " (LÉZY, 2008: 11) puisqu'il est susceptible de transmettre des maladies contagieuses. Ce qui semble l'intéresser davantage c'est leur mode de vie, les outils qu'ils utilisent, leurs ustensiles de cuisine ou les instruments qu'ils jouent, tel un « haltère à boules creuses contenant des graines dures et que l'on agite en cadence » (CREVAUX : 163). Les personnages du Superbe Orénoque sont curieux de leurs mœurs et croyances:

Aussi longtemps que les Barés seront les Barés, l'apparition de ces énormes feux follets au sommet du Duido devra être considérée dans le pays comme un funeste présage, avant-coureur de catastrophes. Aussi longtemps que les Mariquitares seront les Mariquitares, ce phénomène sera pour eux l'indication d'une série d'heureux événements. Ces deux tribus indiennes ont donc une façon très opposée d'envisager les pronostics de leur prophétique montagne (VERNE).

7 Et Crevaux se penche sur leurs parures : chez les Indiens Yarouros, il aperçoit « quelques vieilles femmes dont la lèvre inférieure est hérissée de cinq épingles également espacées dans le tiers médian de la ligne qui joint la peau à la muqueuse. [...] Ces dames ont absolument refusé de me confier le but de cette ornementation »(CREVAUX : 170-171). En allant plus loin, il demande aux Yarouros de danser et tente de les soudoyer :

il y aura du café, du sucre et de la cassave à leur disposition [...] enfin une demidouzaine d'hommes se décident [...] ils se sont fixé sur la tête trois longues plumes d'aras [...] La scène est superbe, par ce clair de lune un peu voilé. Les guayaucos et les bandeaux qui ceignent les fronts se détachent en blanc sur ces silhouettes noires qui offrent un aspect méphistophélique [...] Les femmes accroupies semblent des sorcières attendant leur tour de prendre part au sabbat. Ajoutez le feu dans des poteries où des démons rougis par les flammes font bouillir des herbes magiques ( ibid. : 171-172).

Lexèmes locaux et chromatiques mettent en scène une image inquiétante, voire diabolique, bien qu'admirative des autochtones soulignée par l'impératif adressé au narrataire. Avant de partir, Crevaux achète des objets utilitaires des Indiens qu'il nomme " objets ethnographiques » et Lejanne, son compagnon de voyage, fait, à son habitude, quelques croquis d'Indiens (ibid. : 172). Plus tard, au delta de l'Orénoque, Crevaux fera des moules de pieds, de mains, de tête des Indiens. Si le but exprimé c'est la recherche scientifique dans un territoire où "l'anthropologie [...] trouverait largement son compte " (ibid. : 144), les populations locales sont réifiées, vues comme un objet «à découvrir ». À travers les descriptions, dans un registre anthropologique, les Indiens sont réduits à leur aspect spectaculaire et enfermés dans leur identité de "sauvage » et de "primitif». D'ailleurs les deux auteurs ont la même image de l'Indien nonchalant et fainéant. En effet, Crevaux s' « étonne réellement qu'avec un sol si riche, les habitants de l'Orénoque soient encore si misérables. Il ne faut en chercher la raison que dans leur indolence et leurs faibles besoins » (ibid. : 177). Il en va de même de «la grande paresse que Jules Verne attribue aux indiens banivas » (MANARA, 1998:25), participant ainsi tous les deux à la construction d'une image stéréotypée des Indiens jugés, évalués à travers les critères des Occidentaux. En réalité, les Indiens sont souvent terrorisés par la présence de 
l'expédition qu'ils tentent souvent d'éviter, craignant les maladies et les trafiquants. Crevaux mentionne que lorsque leur embarcation croise un voilier,

[u]n des hommes qui le montent, ne nous ayons pas aperçus, Lejanne et moi, abrités sous le rouf, et croyant sans doute en imposer à nos Indiens, hèle notre canot d'un ton si impérieux que nous défendons de lui répondre. Il renouvelle sa question en nous menaçant de son plomb (CREVAUX : 176).

Les populations locales sont menacées, malmenées, humiliées, alors que les Blancs et les compatriotes de Cravaux « tiennent le haut du pavé dans cette ville vénézuélienne » (ibid. : 185) au bord de l'Orénoque, Bolivar. Les Indiens se voient souvent obligées de vendre de la nourriture qu'ils ont chassée ou cueillie contre des pacotilles, se montrent alors hostiles ou bien «enchantés de savoir qu'on leur apporte des haches, des sabres, des couteaux, des colliers [...] Ils seront contents de la présence des Blancs, jusqu'au moment, il ne faut pas se le dissimuler, où ils auront en leur possession les objets qu'ils convoitent» (ibid.: 203). Ainsi Crevaux semble n'être plus «le civilisateur, le sorcier blanc, le demi dieu, mais un trafiquant de plus », et son " passage comme celui des autres Blancs reste inscrit plus profondément et plus douloureusement dans la chair des populations rencontrées " (LÉZY : 13,11). Ce n'est donc pas à la véritable rencontre de l'habitant de l'Orénoque que vont explorateurs et aventuriers, mais ce qui les motive relève plutôt de leur propres aspirations. Il s'agit pour Crevaux, comme nous l'avons vu, d'une recherche scientifique. En effet, muni d'instruments tels que la lunette micrométrique, le télémètre ou le théodolite, il pratique des essais hypsométriques, mesure la largeur du fleuve, la pression atmosphérique, la température. Il transcrit ses mesures : «nous atteignons le sommet de la Cordillière; les eaux que nous rencontrons maintenant se dirigent vers l'Orénoque! Nous nous trouvons à une altitude de mille neuf

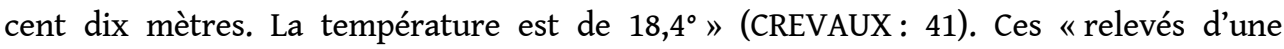
précision exotique» (LÉZY : 8) ainsi que le discours exclamatif rendent compte du dépaysement et de l'étrangeté de l'environnement. Un peu plus tard, avant d'aborder la navigation sur le fleuve Goyabero, jamais exploré auparavant, il s'extasie : « nous saluons la rivière dont nous voulons faire la conquête pour la Géographie !» (CREVAUX : 46). À travers l'épiphonème et le discours militariste, l'espace fluvial apparaît comme un terrain de recherche, un laboratoire à ciel ouvert mais aussi comme un espace à posséder. Comme le remarque E. Lézy, il s'agit plutôt d'une "géographie "héroïque" d'un Ulysse colonial» (LÉZY : 2). Dans Le Superbe Orénoque, si les motivations des deux explorateurs français et de trois géographes vénézuéliens sont similaires, à savoir de découvrir les sources de l'Orénoque, celle du personnage principal sont plus intimistes. Il s'agit pour Jean de Kermor qui est en réalité une jeune fille, Jeanne de Kermor, de retrouver son père disparu aux sources de l'Orénoque. Le récit aboutit à une scène de reconnaissance et de révélation d'identité. Comme le signale J.P. Dekiss, c'est un «parcours à contre-courant d'un personnage travesti » (DEKISS, 1998: 18). Au bout de maintes aventures et épreuves, le personnage regagne son identité, mais non pas celle d'une jeune fille, mais celle d'une femme. Et il n'y aura plus «de retour possible à l'enfance» (ibid.). Cette "anabase marche vers l'intérieur des terres » (LEPAGNEZ: 37) ainsi que l'espace parcouru sont le lieu d'une renaissance et d'une transformation.

En ce qui concerne l'écriture, l'œuvre de Crevaux correspond à un récit d'exploration classique. L'on y retrouve deux cartes géographiques qui retracent son trajet depuis la Cordillière des Andes jusqu'à l'embouchure de l'Orénoque du 17 septembre 1880 au 28 janvier 1881. Quant à la narration, le déroulement de chaque journée est relaté : l'avancée au fleuve, la chasse et la recherche de nourriture, les incidents mineurs ou majeurs. Ainsi 
le 12 janvier il note que «la navigation commence à présenter de grandes difficultés à cause de la brise", le 22 janvier que son compagnon de voyage François Burban est "piqué aux deux pieds par une raie » et un peu plus tard, le 25 janvier que «François Burban est mort en véritable marin, au milieu de la tempête " (CREVAUX : 172-173, 181, 183). Au bout de son périple sur l'Orénoque, il affirme que «c'est avec un véritable enthousiasme que nous saluons l'Atlantique après un voyage de cent soixante jours à travers le continent » (ibid. : 187). La datation calendaire marque et rythme le récit. Selon P. Ricœur, la " databilité se rattache au "compter le temps" »; tout événement est datable "dès lors qu'il est repéré par rapport à "maintenant" ", marquant ainsi "le caractère extatique du "maintenant", c'est-à-dire son appartenance au réseau de l'à-venir, de l'avoir-été, du rendre-présent, et son caractère d'horizon, c'est-à-dire la référence du "maintenant que" aux entités rencontrées dans le monde " (RICCEUR, 1983: 151-153). Dans l'écriture du récit En radeau sur l'Orénoque, la datation indique donc un être-là, un voyageur qui avance au fil du temps et du fleuve ainsi que ce qu'il croise dans son périple. La linéarité de l'écriture diariste et son caractère factuel ainsi que le présent de la narration s'inscrivent dans l'écoulement du temps et le débit continuel du fleuve. L. Britto Garcia transcrit la mythologie des Indiens Karinas à propos de l'Orénoque :

Le fleuve fut fait par Amalivacá et son frère Uochi. Les créateurs eurent de longues discussions pour savoir comment réussir à diriger le courant dans les deux sens pour que les rameurs puissent le remonter sans effort. Comme il trouva cela impossible, Amalivacá prit un canoë pour s'en retourner de l'autre côté de la mer d'où il était venu. [...] Dès lors, le fleuve ou peut-être le temps, coule dans un seul sens (BRITTO GARCIA, 1998 : 11-12).

11 Et il affirme: «le fleuve c'est le temps » (ibid.: 12). C'est le fleuve qui rythme les temporalités ainsi que l'avancée et, par conséquent, celle du récit. Le fleuve est donc un acteur du récit mais aussi un adjuvant et opposant à la fois. Jusqu'à son delta qui marque la fin de la descente. Et Crevaux de signaler : «En revoyant les flots bleus de l'Atlantique, j'éprouve comme une sensation de délivrance. Il semble que je viens d'échapper à un cauchemar, rempli d'émotions tantôt douces, tantôt terribles " (CREVAUX : 192). Après coup, l'Orénoque est donc synonyme d'horreur et d'épouvante. Quant à la narration vernienne, elle suit le même développement linéaire, bien qu'en sens inverse : c'est une remontée et non pas une descente. En tant que roman d'aventures classique, il mélange narration, analyse et dialogues, trois éléments caractéristiques du genre (TADIÉ, 1996: 57). Roman-fleuve à plus de six cents pages, Le Superbe Orénoque suit aussi l'ordre chronologique qui s'étend de juillet 1892 à février/mars 1893, hormis quelques rares digressions nécessaires à la compréhension de la situation présente (LEPAGNEZ : 43). Un chapitre en est particulièrement symptomatique, le premier de la seconde partie, au titre fort explicite : Quelques mots du passé (ibid.). Par ailleurs, Verne mentionne le « chubasco ", le «terrible coup de vent » (VERNE) qui se déchaîne sur le lit de l'Orénoque et fait tomber Jean de Kermor de l'embarcation. Un des explorateurs français, Helloch plonge dans le fleuve et lui sauve la vie, tout en découvrant son identité féminine. Crevaux de sont côté relate que vers « le milieu du fleuve, des vagues hautes et serrées prennent obliquement notre canot et le balancent comme un bouchon. Dix fois nous avons failli chavirer. Quelques hideux caïmans montrent leur crête dorsale à fleur d'eau et ajoutent quelque horreur à notre situation déjà si lugubre » (CREVAUX: 182). Un d'eux a déjà failli engloutir Apatou ce qui retarde le prochain départ. Dans les deux œuvres, le fleuve, avec ses phénomènes naturels, est donc un véritable agent actantiel du récit. 
12 Moravagine, de Blaise Cendrars met en scène le personnage de Raymond, médecin psychiatre qui libère, lâche dans la nature un "grand fauve ", Moravagine. Après avoir parcouru la Russie pré-révolutionnaire, participant à la lutte violente du mouvement nihiliste, après un passage par Berlin où Moravagine commet des féminicides en série et un passage par les États-Unis à l'époque de la ruée vers l'or, leur périple passe par la descente du fleuve Orénoque. Dans Remonter l'Orénoque, de Mathias Enard sont relatées deux narrations parallèles : celle d'une femme, d'une infirmière qui remonte l'Orénoque tout en cherchant à connaître le Venezuela, pays d'origine de son père qu'elle a très peu connu. Et puis celle d'un médecin vénézuélien en France qui suit l'histoire amoureuse et la descente aux enfers d'un couple d'amis et collègues, un médecin et de la narratrice qui est infirmière, dont il est secrètement amoureux, en même temps que le débordement des urgences de l'hôpital lors d'un été caniculaire. Nous examinerons les descriptions, le récit ainsi que les éléments intertextuels afin d'identifier les enjeux du voyage contemporain sur l'Orénoque et les images du fleuve.

13 Lors de leur descente de l'Orénoque, les deux personnages principaux de Moravagine voient qu'« à perte de vue, la rive ne formait qu'un immense rempart chaotique, forêts renversées, racines, broussailles dénouées, trous, cratères boueux, plaies béantes, éboulis, grands pans de terreau noir glissant à l'eau » (CENDRARS : 164). La topographie du paysage tropical rend compte d'une nature désordonnée et dévastée, soulignée par l'énumération, qui fait en quelque sorte l'économie du tumulte que traversera le personnage de Raymond. La description de la flore est toujours présente :

Une végétation folle, basse, immergée, reluisante, inextricable envahissait l'étendue. Au fond de l'horizon, un pli sombre marquait la forêt vierge, la forêt tropicale. C'était la terre ferme. Nous nous engageâmes dans une multitude de canaux, nous suivîmes de nombreux méandres et parcourûmes tout un réseau de sinuosités, chenaux, goulets, passes, rigoles pour déboucher tout à coup sous le dôme de la haute forêt. C'était majestueux et inattendu. Nous nous trouvions au milieu du fleuve (ibid.).

14 La juxtaposition d'adjectifs produit un effet de profusion végétale, faisant de l'Orénoque un espace de démesure. De surcroît, l'énumération des cours d'eau rend compte de la diversité géographique fluviale où nous retrouvons l'image d'un fleuve imposant, grandiose et surprenant, déroutant à la fois, un espace exceptionnel. C'est cette image de dédale qui est présentée également dans Remonter l'Orénoque, "une toile d'araignée de cours d'eau sauvages, de rivières striant les forêts pour rejoindre au hasard les entrelacs amazoniens » (ENARD, 2015 : 47). La métaphore arachnéenne ajoute un aspect effrayant, sinistre à l'image du fleuve. Plus tard, Raymond et Moravagine descendent vers le bas l'Orénoque où « il y a beaucoup de plantations, - café, cacao, canne à sucre ; il y a surtout des plantations de bananiers. Elles s'étendent durant des semaines sur les rives inégales du fleuve » (CENDRARS, 1926 : 164-165). La flore est toujours présente dans la description, cependant d'une part elle est énumérée, passée rapidement, d'autre part, il ne s'agit plus d'une flore «sauvage » et exotique, caractéristique de la végétation de l'Orénoque, mais des plantations, des exploitations agricoles des bords fluviaux. En outre, la perception du temps se modifie, les mesures temporelles étant utilisées pour compter l'espace. Perturbation de la perception de l'espace, perturbation de la perception du temps; le malaise s'installe de plus en plus et le narrateur relate:

Nous étions maudits. La nuit ne nous apportait aucun repos. Dans la brume bleuâtre du soir qui succédait à la pluie, des milliers de végétaux aux panaches plumeux s'égouttaient. D'immenses chauves-souris se laissaient choir. Des cascabelles 
ondulaient entre deux eaux. L'odeur musquée des crocodiles nous soulevait le cœur (ibid. : 168).

La faune tropicale inspire donc toujours la crainte, la menace, voire le dégoût, le malaise et l'Orénoque devient un espace trouble, angoissant. Quant à Joanna, la narratrice de la remontée de l'Orénoque, elle imagine dans sa cabine les «couleurs des oiseaux fantastiques que je chasserai du regard dans les frondaisons, ou la tendresse fugitive des singes, des tamanoirs et des lynx que je ne verrai sans doute pas mais dont je sentirai la présence, toute proche, une fois passées les plaines » (ENARD : 48). Tout l'imaginaire de la faune multicolore exotique est là, pourtant cette faune n'est pas vue, décrite mais juste connue d'avance et imaginée. Aucun contact visuel avec celle-ci, ce qui n'est pas le cas des personnages de Moravagine :

Nous nous dissimulions, nous nous tassions entre les racines en caoutchouc qui viennent s'arc-bouter sur les rives comme les pattes fantasques de quelque monstrueuse tarentule. Nous dormions d'un sommeil agité. Lycanthropie. Celui dont c'était le tour de garde résistait de son mieux à l'envoûtement des moustiques en imitant les longs miaulements des guépards. Au ciel, la lune enflait comme une piqûre. Les étoiles rougissaient comme les traces apparentes d'une morsure (CENDRARS : 168).

Les personnages semblent ne former plus qu'un avec la nature, de faire partie de celle-ci, de se transformer eux-mêmes en animaux sauvages. L'espace fluvial devient un lieu de métamorphose et de zoomorphisme. En outre, les métaphores animalières qui sont utilisées pour décrire la paysage, la référence à l'agression physique font que l'Orénoque garde son image d'un espace de terreur et de souffrance. De la même manière, dans Remonter l'Orénoque, le fleuve est décrit comme une "longue blessure sur la carte », une «blessure d'eau vive qui balafre son pays » (ENARD : 47, 50). En allant même plus loin, il est qualifié de "veine cave du Venezuela, cette aorte». La métaphore médicale renvoie bien entendu à l'identité professionnelle de la narratrice, Joana, mais en plus la référence à l'anatomie du cœur fait référence à l'enjeu affectif dont le fleuve devient l'espace et le symbole. Un espace qui est pourtant ambigu. La narratrice se demande : «Où commence la mer, dans l'immensité du delta ?» (ibid. : 31), et Raymond exprime les mêmes doutes : "On ne sait pas exactement où est la limite de l'eau douce et de l'eau salée, ni où commence la terre, ni où finit la mer ». L'espace deltaïque semble être un espace sans frontières, sans contours et par là inquiétant, ce qui est souligné par l'interrogation. Dans Moravagine, l'Orénoque garde son aspect épouvantable et effrayant :

Tout devenait monstrueux dans cette solitude aquatique, dans cette profondeur sylvestre, la chaloupe, nos ustensiles, nos gestes, nos mets, ce fleuve sans courant que nous remontions et qui allait s'élargissant, ces arbres barbus, ces taillis élastiques, ces fourrés secrets, ces frondaisons séculaires, les lianes, toutes ces herbes sans nom, cette sève débordante, ce soleil prisonnier comme une nymphe et qui tissait, tissait son cocon, cette buée de chaleur que nous remorquions, ces nuages en formation, ces vapeurs molles, cette route ondoyante, cet océan de feuilles, de coton, d'étoupe, de lichens, de mousses (CENDRARS : 166).

L'énumération ludique qui met ensemble des éléments aussi dépareillés que des ustensiles, des gestes, de la nourriture, fait ressortir le caractère de désordre menaçant de l'espace fluvial. L'énumération accumulative ascendante des éléments naturels qui s'ensuit, met l'accent sur l'abondance de ce désordre, sur son caractère luxuriant et excessif. La juxtaposition est indicative de la diversité visuelle qu'offre l'espace orénoquien. C'est un tout autre désordre que présente le fleuve à l'époque actuelle :

À Santo Tomé de Guayana, à Puerto Ordaz, les longs minéraliers s'y avancent comme sur la mer. Puis aussi bien les rondins qui descendent le Grand Fleuve, les 
diamants arrachés à ses rives, l'or extrait de ses alluvions, les bacs qui le traversent que les barcasses qui le remontent, tous semblent nager dans une mer de boue suée par la verdure de la plaine, trouée par instants d'une usine, d'une station de pompage (ENARD : 146).

L'accumulation rend compte de l'exploitation d'économie primaire du fleuve qui devient un lieu presqu'industrialisé. Plus de navigation éprouvante, mais une multitude d'embarcations qui le naviguent, le traversent dans tous les sens faisant un lieu de circulation commerciale. Point de flore et de faune exotiques, de nature sauvage; l'apprivoisement, voire le « développement » sont de mise. Le port lui-même est :

un lieu de l'existence duquel on pourrait presque douter s'il n'y avait les mouvements des cargos, des barges, des porte-conteneurs et des grues qui les chargent, de l'aube au couchant dans un ballet besogneux. Les longs minéraliers [...] les remorqueurs virevoltent, les conteneurs multicolores qui s'empilent sur la berge ne laissent rien deviner des richesses qu'ils renferment, le café, les fruits, les conserves, l'or, les armes, le rhum ou la cocaïne s'y cachent sans qu'aucun parfum s'en exhale et les seules embarcations odorantes sont les volaillers, dont les innombrables cages de métal dissimulent mal les cris effrayés des poulets et répandent une puanteur de cloaque en guise de fumée (ibid. : 13-14)

L'énumération qui semble être la figure de description du fleuve par excellence, insiste sur l'espace commercial qu'est devenu celui-ci, espace de trafics légaux et illégaux dans une économie mondialisée. Espace d'échanges et de transactions d'où la faune sauvage est absente, remplacée par des animaux domestiques enfermés, objets marchands. Pas de caïman qui surgit et dévore comme celui qui a blessé Apatou dans le récit de Crevaux que la narratrice lisait passionnément dans son enfance. Joanna était d'autant plus impressionnée par la gravure qui représentait la plaie béante d'Apatou dont « la légende sous l'illustration disait, tout simplement, Plaie d'Apatou, ni plus, ni moins, on sentait que les explorateurs allaient en profiter pour mesurer la taille de la denture, l'écartement des mâchoires de la bête, directement sur la peau d'Apatou le Noir» (ibid.: 131-132). L'élément intertextuel est repris sous un point de vue postcolonial qui montre la réification $\mathrm{du}$ Noir, sa réduction en animal de laboratoire au nom de l'avancée scientifique. C'est cependant cette image et cette lecture qui forgeront chez la narratrice l'image du pays de son père absent et qui susciteront chez elle l'envie de soigner, de guérir ce qui déterminera son avenir professionnel. L'envie de connaitre un pays motivée par les lectures est une constante de l'écriture du voyage et les fragments intertextuels d'un récit de voyage à l'autre sont fréquents. La narration de Moravagine effectue également des « emprunts à des ouvrages de voyageurs ou de géographes comme Élisée Reclus » (TOURET, $2000 ; 180$ ). Ces emprunts concernent autant les descriptions des éléments naturels et géographiques que les reprises thématiques. Raymond et Moravagine se font prisonniers des Indiens Bleus, ce qui fait référence à toute une littérature de récits de captivité des voyageurs et des explorateurs par des "sauvages ». M. Touret note que les reprises des récits des grands voyageurs par les œuvres de fiction assurent «une continuité entre le récit des premiers conquérants de nouveaux territoires, les relations ethnographiques et les entreprises séduisantes et romanesques de la quête de mondes lointains ou perdus » (TOURET, 2006: 168-169). Dans Moravagine, nous retrouvons donc le registre anthropologique avec des prosopographies des Indiens Bleus, des éléments relatifs à leur mode de vie et les coutumes, leur habillement et parures et leur domiciles, leur langues et leurs croyances. Ces dernières comportent la fête du « Jeune Homme Pénitent » qui est : 
habillé avec magnificence. Des parfums brûlent sur son passage, on répand le sang des animaux, on lui présente des fleurs, des fruits et des graines. [...] Partout la foule se prosterne pour l'adorer, car il est l'image vivante, l'image humaine du soleil. Non seulement il mène durant un mois joyeuse vie, toutes les cases lui sont ouvertes, on lui prépare les meilleurs mets [...] mais encore il épouse publiquement quatre jeunes vierges d'une rare beauté qui lui sont spécialement destinées (CENDRARS : 174-175). l'image du Blanc vénéré par les « sauvages » comme un demi-dieu, très fréquente dans les récits de voyage et d'exploration. D'ailleurs ce n'est pas sans une certaine ironie que les aspects anthropologiques de la population locale sont décrits. Nous y reviendrons. Dans le cadre du registre anthropologique est relatée également l'histoire des Indiens Bleus qui « appartenaient à l'antique tribu des Jivaroz. Avant la conquête, les Jivaroz étaient toutpuissants. D'un tempérament guerrier, ils étaient toujours en luttes avec leurs voisins les Sutagaos et les Tunjas ; depuis la conquête, leur nombre a considérablement diminué » ( ibid. : 172). Comme le remarque L. Guyon, par leur force et leur sauvagerie, ils appartiennent «à la race des hommes tropicaux, telle que la définit Nietzsche, et l'adaptation des personnages du roman à leurs mœurs manifeste un accord profond de l'Homme avec la Vie, dans sa dimension la plus farouche et la plus originelle » (GUYON, 1998: 51). Bien que la considération nietzschéenne puisse paraître obsolète, l'accord entre l'homme et la nature jouera un rôle important dans la séquence orénoquienne. C'est aussi à l'histoire des Indiens que fait référence Joana, ces Indiens qui « sont l'origine toujours présente du monde, semble-t-il, ils n'ont pas d'histoire et pas de mémoire, à ce qu'il paraît » (ENARD : 134). Elle opère ainsi une projection, au sens psychanalytique, de l'abence paternelle de son histoire familiale sur l'histoire perdue, effacée des Indiens. Elle, qui n'a que très peu de souvenirs de son père, s'identifie à ceux qui ont été coupés de leur mémoire collective, tissant ainsi un lien d'affinité et de communauté avec eux.

Par ailleurs, les personnages des deux œuvres sont souffrants. Joana souffre de la chaleur au même temps que dans la narration parallèle, la canicule sévit à Paris, et est sujette à des nausées et des vomissements qui lui font penser à une éventuelle grossesse. Tandis que Raymond est victime de fortes fièvres et presque dans un état comateux : «les jours passaient. Les jours, les nuits. Tout m'était absolument égal» (CENDRARS : 176-177), perdant la notion du temps, ce qui est rendu par la phrase courte et son rythme bref. Il semble aussi ressentir des «milliards d'éphémères, d'infusoires, de bacilles, d'algues, de levures, regards, ferments du cerveau. Silence » (ibid. : 166). L'énumération désordonnée met en scène les aspects fragmentaires de la perception tout en insistant sur le nombre et la variété des éléments nocifs. L'Orénoque garde ainsi son image d'espace de maladie et de souffrance.

Si la maladie tropicale est aussi une constante des récits de voyage d'autrefois où les voyageurs narrent leurs mésaventures, dans les deux œuvres contemporaines son enjeu est tout autre. Lors de ses accès de fièvre, Raymond déclare : «J'avais tout oublié. [...] Je n'ai pas pensé une seule fois à l'Europe ni au moyen de rentrer dans les pays civilisés. Qu'est-ce que tout cela pouvait bien me faire? J'avais tout oublié» (ibid.: 177). La traversée de l'Orénoque intervient dans le récit juste après une équipée aux États-Unis où il est question du «principe de l'utilité » qui anime le monde et s'ensuit le retour en Europe. La séquence "sauvage » se situe au milieu de la narration du monde « civilisé ». Le personnage de Moravagine adhère pendant un moment aux mœurs des Indiens. Pour l'Européen qu'il est, «les rituels totémiques d'un autre âge rendent un semblant 
d'identité » (FLÜCKIGER, 1978, 193). Par cette adhésion ainsi que par la monstruosité décrite du monde « civilisé » dans l'œuvre, ce dernier ainsi que ses avancées, ses systèmes et ses classes sont remis en question. En effet, «Moravagine contient une longue et virulente critique de la petite bourgeoisie. Le cadre de la vie urbaine, mesquine et faussement civile, les lieux et les insignes de la vie des citoyens dans un régime de démocratie politique [...] l'idéal de la petite propriété individuelle sont ici stigmatisés » (TOURET, 2000: 188). Loin de venir en "civilisateur", l'enjeu de la navigation de l'Orénoque pour les personnages est donc, en grande partie, l'éloignement, l'échappée d'un monde policé. C'est s'évader de son monde, de sa vie qu'est le but du voyage de Joana aussi. Elle se dit : « voilà mon monde à présent, avec pour seule ouverture ce hublot à un mètre cinquante de la surface, noyé dans les reflets verdâtres du fleuve qui viendra, dès la première tempête, se lancer à l'assaut du plat-bord et frapper avec une force digne d'un océan le verre sali, au pourtour rouillé, qui protège ma fuite » (ENARD : 14). S'échapper du monde, le tenir à distance à travers le hublot de la cabine, telle est la motivation de son voyage. Son exil, "ni vain ni glorieux", consistera à se "mélanger aux boues de l'Orénoque et retrouver mes racines, comme on dit, elles ne sont qu'un champ de ruines fertiles où poussent les pierres précieuses, lointaines et anonymes, de tant d'humains mêlés » (ibid. : 107). Loin des exploits des grands navigateurs et explorateurs, le traversée de l'Orénoque prend pour la narratrice la forme d'un enjeu personnel. Il s'agit davantage d'un voyage intérieur que d'un déplacement géographique. L'Orénoque prend alors l'allure d'un espace d'accomplissement personnel où l'enjeu se trouve à l'intérieur plutôt que dans le monde extérieur. C'est le cas également de Raymond qui, sujet à la fièvre et au délire, tombe dans son espace intérieur. Il délire : «Je n' avais aucune inquiétude, aucun souvenir. Rien, rien, rien. Rien que de la fièvre. Une fièvre lente. J'étais dans un état fondant, avec du velours sous la peau. Paludisme. J'étais abruti, idiot, sans pensée, veule. Sans pensée, sans passé, sans futur. » (CENDRARS : 177). Cet égarement presque comateux embrasse de multiples enjeux. $\mathrm{Si}$, comme le signale $\mathrm{C}$. Leroy, la description «évoque l'amputation » (LEROY, $1998: 79$ ), cette séquence descriptive renvoie à l'auteur lui-même et à son œuvre. En effet, d'après C. Le Quellec Cottier :

Après la mutilation de 1915, Cendrars est un homme détruit, défait. Il rejette fondamentalement cette Europe déchue qui a traîné l'humanité dans la boue et cherche, par l'art, à dépasser les codes d'une société qu'il considère décomposée. Pour cela, il se tourne vers l'art nègre, expression première d'une altérité découverte par les artistes dès le début du siècle, et à travers cet univers, exploite un imaginaire primitif qui ne doit rien à l'Occident. Cette impulsion "nègre " facilite la réapparition de Moravagine, qui a, dès 1917, commencé à prendre forme en tant que roman éclaté, exprimant ainsi son rejet du monde bourgeois qui a conduit à la faillite une société dégénérée (LE QUELLEC COTTIER, 2006 : 114).

Il s'agit donc de fuir un Occident de plus en plus rationalisé et industrialisé, devenu réellement sauvage, afin d'aller vers le naturel, voire le "primitif», d'une société mécanisée et bruyante vers l'apaisement. Raymond affirme n'avoir « aucune inquiétude, aucun souvenir. Rien, rien, rien. Rien que de la fièvre. Une fièvre lente. J'étais dans un état fondant, avec du velours sous la peau. Paludisme. J'étais abruti, idiot, sans pensée, veule. Sans pensée, sans passé, sans futur " (CENDRARS : 177). Le personnage se trouve dans un état extatique de quiétude et d'impassibilité à la fois. La réitération de "rien " marque aussi bien l'étourdissement et la perception réduite que son ataraxie. Pour lui, « tout était éternel et pesant. Lourd. Le soleil. La lune. Ma solitude. La nuit. L'étendue jaune. Les brouillards. La forêt. L'eau. Le temps pipé par un crapaud ou une ultime gaguère : do-ré, do-ré, do-ré, do-ré, do-ré, do-ré... Inattention. Indifférence. Immensité. 
Zéro, Zéro d'étoiles. On appelle ça la Croix du Sud. Quel sud ? Zut alors pour le sud. Et le nord. Et l'est et l'ouest et tout. Et autre chose. Et rien. Merde» (ibid.). Encore une énumération ludique qui vire vers l'absurde, indicative paradoxalement à la fois du marasme et de l'excès. Les onomatopées signalent une vision désordonnée du monde extérieur à travers l'état fiévreux, tout en rythment la phrase. Cependant les contrastes, géographiques, conceptuels, marquent une unité, celle de « devenir le Grand Anonyme, l'un des désirs insistants, inassouvis de la pensée de Cendrars » (FLÜCKIGER, 2003, 353). Le discours bref, saccadé traverse différents aspects et impressions de la réalité. Et en même temps, le personnage «se délivre enfin de la masse commune du langage, comme du chaos procède le cosmos " (LEROY : 79). La narration opère alors un sommaire qui prend fin avec l'arrivée de Moravagine après avoir été adulé par les Indiens. Raymond « écoute. Une nuit que j'étais étendu sur ma couche, on m'appela par mon nom. Quel nom ? Étais-je encore vivant? On avait murmuré mon nom, mon petit nom, Raymond. Étrange, je n'arrivais pas à comprendre " (CENDRARS, 177-178). Moravagine le rappelle à la vie en prononçant son nom, par «la puissance des syllabes qui le raniment en le renommant " (LEROY : 79). Il lui raconte comment il s'est échappé aux Indiens et a tué ses épouses, prenant sa propre voix narrative à la première personne et couvrant ainsi le sommaire du récit de la part du Raymond-narrateur. Celui-ci revient à la vie après « une sorte de descente aux enfers » (TOURET, $2000: 108)$. Cette envie de renaissance à travers le retour aux sources, le retour vers l'enfance traverse aussi Joana. Dans le pays de son père, des racines familiales, à bord du bateau, elle s'« accroche malgré la douleur et le mal de mer à ce bateau qui m'emmène dans le Sud pour tout recommencer, renaître, effacer, reprendre, remettre à neuf, dans un pays vierge et nu, mon existence " (ENARD, 118). Il s'agit d'un retour aux sources, d'une catabase vers le passé afin de se tourner à nouveau vers l'avenir. Ce que le personnage semble réussir :

Voilà, je suis enfin moi-même, dans ce réduit comme une cale, bercée par le hublot ouvert sur l'eau tourmentée, boueuse et verdâtre, toute illusion, tout mirage emportés par le courant, vers le delta marécageux, comme le sang s'enfuit, me quitte en tremblant, je le sens fluer entre mes jambes par à-coups, c'est le cycle qui reprend, l'ordre des choses, et la tristesse qui pourrait m'envahir, la perte, n'est qu'un fluide de plus laissé dans le fleuve, le contenu d'un estomac précipité audehors par la nausée, un récit qui s'efface. Il est difficile d'aller vers la source, il faut se défaire de tant de poids. S'alléger au maximum pour profiter des paysages, des cataractes, comme les Indiens vont, eux, presque nus dans la nature (ibid. : 137).

Si la description de la faune et la flore, des paysages se trouve très réduite, cela ne correspond pas à l'indifférence ou à l'invisibilisation; c'est parce que l'enjeu du voyage n'est pas extérieur, mais intérieur. La nudité des Indiens ne renvoie à un aspect "primitif ", mais au dépouillement de tout, y compris le passé pesant. C'est ce qu'indique par ailleurs le vomissement, acte d'extériorisation par excellence, qui marque l'expulsion corporelle de tout élément nocif et, par extension, de tout ce qui appartient au passé. Toutefois, cela semble irréalisable : « c'est impossible de tout recommencer, impossible de se défaire de soi, de reconstruire, dans des bras neufs, une existence en cours de destruction, on est trop loin, le bateau est déjà parti, la rive éloignée, il ne reste plus que le long mystère de l'Orénoque, où rien ne se perd de ce qui aurait pu être, car j'emporte tout avec moi» (ibid.: 139). Ce qui met en exergue l'irréversibilité du passé et l'impossibilité de remonter le temps, de remonter aux sources. L'Orénoque revêt donc des images opposées, celle de la catabase et de l'anabase, de l'espace de la renaissance et de son impossibilité. 
24 En ce qui concerne la narration, dans Remonter l'Orénoque, une œuvre à deux voix, tout le récit d'Ignacio est une longue digression vers le passé, une tentative de raconter, de remonter aux sources de l'histoire amoureuse de Joana avec le médecin Youri. À son flux des souvenirs, s'entremêle le récit de la remontée de Joanna dont les souvenirs renvoient à différentes époques de sa vie par une écriture de flux de conscience qui n'est pas sans évoquer le flux du fleuve lui-même. Les deux récits, les phrases « s'amalgament, s'étalent et font delta " (HOUSSIN, 2005). Quant au récit cendrarsien, si la narration est linéaire, prise en charge par la personnage de Raymond, il n'en va pas de même de la focalisation. Dans la séquence de la maladie et de l'évasion, son rôle de narrateur s'amoindrit, voire s'efface. Presqu'anéanti physiquement, sa voix disparaît également. C'est par la voix de Moravagine, par une analepse complète interne, une anachronie homodiégétique que le massacre des ses épouses Indiennes et le plan d'évasion sont relatés. Ces « aventures focales du narrateur Raymond » (FLÜCKIGER, 1978, 232) nous renvoient au caractère de roman d'aventures que revêt Moravagine et sa séquence orénoquienne. Cependant les aventures que vivaient autrefois les voyageurs et les explorateurs sont presque caricaturées. Les constantes du récit de voyage classique, telles que la captivité, la maladie, l'homme Blanc vu comme un demi-dieu sont parodiées. L'ironie avec laquelle les aspects anthropologiques de la population locale sont décrits, comme nous l'avons vu, ainsi que la manière extravagante dont les aventures des deux personnages sont narrées et leur auto-dérision renforcent le registre parodique. Apparaît ainsi une image de l'Orénoque qui a perdu son caractère d'espace de véritables péripéties et d'aléas, d'espace de découverte. Quant au récit enardien, c'est par la focalisation d'Ignacio que le narrataire apprend l'agression physique qu'a subie Joana de la part de son compagnon, son hospitalistion en état de coma cérébral et sa mort. Toute la narration de Joana est alors remise en question et semble être une hallucination, une évasion d'un esprit qui s'échappe du corps et qui voyage, qui navigue vers les sources de l'Orénoque. Elle se trouve confirmée ainsi l'impossibilité de toute fuite que ressent la narratrice, de toute remontée vers les sources de l'existence puisque celle-ci ne subsiste plus. La vie n'existe plus tout comme l'Orénoque d'antan qui n'existe plus, puisqu'il est devenu un espace cartographié et connu, un espace exploité au nom du « développement ».

Pour conclure, nous pouvons affirmer qu'au XXIe siècle l'Orénoque n'apparaît plus dans la fiction comme un espace à posséder. Si dans Moravagine, nous retrouvons l'image d'un espace inquiétant qui inspire l'épouvante ou dégoût, ce qui a formé son imagerie au XIXe siècle, un espace de démesure, ceci est largement relativisé par le registre parodique. L'espace fluvial a perdu son caractère d'espace de découverte et d'exploration au profit d'une imagerie qui renvoie à la métamorphose, la renaissance et au retour aux source, un lieu d'évasion. Il revêt désormais le rôle d'un enjeu intérieur et personnel. Et l'image de l'espace orénoquien comporte toujours la souffrance, celle-ci n'est plus physique, dans Remonter l'Orénoque, mais animée par la conscience de l'impossibilité de toute fuite. D'ores et déjà lieu de circulation marchande, l'Orénoque n'est pas invisibilisé dans le récit enardien, mais transfiguré. Ainsi Moravagine et Remonter l'Orénoque, bien que de points de vue différents marquent une rupture avec la vision du fleuve d'avant. 


\section{BIBLIOGRAPHIE}

CENDRARS Blaise, Moravagine suivi de La fin du monde filmée par l'Ange N.-D. et de L'Eubage, présentée et annotée par Jean Carlo FLÜCKIGER, Denöel, coll. Tout autour d'aujourd'hui, 2003 [1926].

CREVAUX Jules, En radeau sur l'Orénoque. Des Andes aux bouches du Grand Fleuve, Paris, Seuil, coll. Points Aventure, 2018 [1882].

ENARD Mathias, Remonter l'Orénoque, Actes Sud, coll. Babel, 2015.

VERNE Jules, Le Superbe Orénoque, Arvensa Éditions, format Kindle, 2015 [1898].

BRITTO GARCIA Luis, « La mémoire de l'Orénoque », dans L'Énigmatique Orénoque, sous la direction de Jean-Paul DEKISS, Revue Jules Verne, N6, 1998, pp.11-15.

DEKISS Jean-Paul, «L'Orénoque, un fleuve au masculin-féminin », dans L'Énigmatique Orénoque, sous la direction de Jean-Paul DEKISS, Revue Jules Verne, N6, 1998, pp.18-21.

LEROY Claude, La main de Cendrars, Presses Universitaires du Septentrion, coll. Objet, 1998.

LÉZY Emmanuel, « “Jules Crevaux, l'explorateur aux pieds nus”. Un mythe géographique amazonien », EchoGéo 7 / 2008, 17.12.2008, URL: http://echogeo.revues.org/9983

FLÜCKIGER Jean Carlo, Au cour du texte : Essai sur Blaise Cendrars, Payot, coll. À la baconnière, 1978.

GUYON Laurence, «Le Double et le surhomme », dans Blaise Cendrars. Sous le signe de Moravagine (tome 6), sous la direction de Jean Carlo FLÜCKIGER, Claude LEROY, Lettres Modernes Minard, coll. La Revue de Lettres Modernes, 2006, pp.43-58.

HOUSSIN Xavier, Mathias Enard, roman fleuve, Le Monde des Livres, 14.04.2005. URL : https:// www.lemonde.fr/livres/article/2005/04/14/mathias-enard-roman-fleuve_638827_3260.html

LEPAGNEZ Claude, «Le Superbe Orénoque, comme impossible anabase », dans L'Énigmatique Orénoque, sous la direction de Jean-Paul DEKISS, Revue Jules Verne, №6, 1998, pp.37-43.

LE QUELLEC COTTIER Christine, "Transparence dans Moravagine ", dans Blaise Cendrars. Sous le signe de Moravagine (tome 6), sous la direction de Jean Carlo FLÜCKIGER, Claude LEROY, Lettres Modernes Minard, coll. La Revue de Lettres Modernes, 2006, pp.113-128.

MANARA Bruno, «Le Superbe Orénoque en 80 mondes ", dans L'Énigmatique Orénoque, sous la direction de Jean-Paul DEKISS, Revue Jules Verne, N6, 1998, pp.23-35.

RICCEUR Paul, Temps et récit II : La configuration dans le récit de fiction, Seuil, 1983.

TADIÉ Jean-Yves, Le roman d'aventures, PUF, coll. Quadrige, 1996.

TOURET Michèle, Blaise Cendrars, le désir du roman, Honoré Champion, coll. Cahiers Blaise Cendrars, 2000.

TOURET Michèle, « À travers le monde, à travers les modèles ? ", dans Blaise Cendrars. Sous le signe de Moravagine (tome 6), sous la direction de Jean Carlo FLÜCKIGER, Claude LEROY, Lettres Modernes Minard, coll. La Revue de Lettres Modernes, 2006, pp.163-182.

WESTPHAL Bertrand, La géocritique : Réel, fiction, espace, Paris, Seuil, 2007. 


\section{RÉSUMÉS}

$\mathrm{Au}$ XIXe siècle, le fleuve Orénoque apparaît dans la littérature comme un espace «sauvage » et inquiétant, dépaysant, un espace de danger mortel mais aussi de l'extraordinaire. Une large part est faite à la description de la faune et de la flore. En revanche, aux XXe-XXIe siècles, des mutations s'opèrent concernant la perception de l'espace fluvial ainsi que son rôle dans la narration. L'espace fluvial semble davantage être un lieu où se joue un enjeu intérieur et le voyage devient quête personnelle. S'il reste un espace de souffrance, celle-ci peut aboutir - ou pas - à une transformation et une renaissance. Le récit enardien, offre l'image contemporaine d'un espace de circulation marchande.

In the nineteenth century, the Orinoco River appears in literature as a « wild " and disturbing, exotic space, a space of mortal danger but also of the extraordinary. Much is made of the description of fauna and flora. By contrast, in the XX-XXI centuries, changes are taking place concerning the perception of the river space as well as its role in the narration. The fluvial space seems to be more of a place where an internal issue is played out and the journey becomes a personal quest. If the river remains a space of suffering, it can lead - or not - to a transformation and a rebirth. Enard's narrative presents the contemporary image of a space of commercial circulation.

\section{INDEX}

Mots-clés : Orénoque; géocritique ; littérature francophone ; littérature comparée

Keywords : Orinoco; geocritics ; French-speaking literature ; comparative literature

\section{AUTEUR}

\section{KAROLINA KATSIKA}

Université de Bourgogne Franche-Comté, Laboratoire CRIT EA3224

Littérature comparée

katkarolina@yahoo.fr 